\title{
Preparation of microgel composite hydrogels by polymer post-crosslinking method
}

\author{
X. P. Qin, F. Zhao, S. Y. Feng* \\ School of Chemistry and Chemical Engineering, Shandong University, Jinan, 250100, P. R. China
}

Received 22 September 2010; accepted in revised form 6 December 2010

\begin{abstract}
A novel post-crosslinking method by heating the composite polymer with dispersed reactive microgels was used to prepare microgel composite (MC) hydrogels. This MC hydrogels were crosslinked by reactive microgels instead of traditional crosslinkers. The reactive microgels contained hydroxymethyl groups, which acted as post-crosslinkers. They were prepared by inverse emulsion polymerization. The formed MC hydrogels at appropriate conditions had high equilibrium swelling ratio and excellent mechanical properties. The heating conditions and the polymer water content influenced the hydrogel properties. For MC hydrogels prepared by direct heating the as-prepared composite polymers with $75 \%$ water content, their properties were influenced by heating conditions. When the heating conditions were $90^{\circ} \mathrm{C}$ for $4 \mathrm{~h}$, the $\mathrm{MC}$ hydrogel had a tensile strength of $32 \mathrm{kPa}$ and a high elongation of $960 \%$. In addition, for MC hydrogels prepared by heating the partly evaporated composite polymers, their properties can be adjusted by the polymer water content. Appropriate reduction of the polymer water content was beneficial to improve the mechanical strength of the MC hydrogels. The appropriate polymer water content was important to prepare MC hydrogels with excellent mechanical properties. When the composite polymer with $50 \%$ water content was heated at $90^{\circ} \mathrm{C}$ for $3 \mathrm{~h}$, the MC hydrogel had high tensile strength of $130 \mathrm{kPa}$ and high tensile elongation of $503 \%$.
\end{abstract}

Keywords: mechanical properties, hydrogels, reactive microgels, post-crosslinking

\section{Introduction}

Polymeric hydrogels have attracted much scientific interest over the past several decades and have found uses in many fields such as superabsorbents, in medicine, hygiene, and in biomedical applications [1]. Given increasing environmental issues, the idea of replacing plastics with hydrogels also seems reasonable [2]. However, the applications of the conventional hydrogels crosslinked by the traditional crosslinkers such as $N, N^{\prime}$-methylenebisacrylamide are strongly limited by their poor mechanical properties. In recent years, several synthesized hydrogels with excellent mechanical properties have been successfully prepared such as topological hydrogels [3], double-network hydrogels [4, 5], and composite hydrogels [6-15]. The composite hydrogels include the nanocomposite (NC) hydrogels using inorganic clay as a crosslinker $[6,15]$, the macromolecular microsphere composite (MMC) hydrogels using hydrophobic macromolecular microsphere as a crosslinker [11, 14], and the hydrophilic reactive microgel (HRM) composite hydrogels using hydrophilic reactive microgels containing $\mathrm{C}=\mathrm{C}$ double bonds as a multifunctional crosslinker [13]. The HRM composite hydrogels are made from hydrophilic organic monomers only and do not require inorganic or hydrophobic fillers. They have a two-level hierarchic network structure, a primary network inside reactive microgels and a secondary network between microgels similar to $\mathrm{NC}$ or MMC

\footnotetext{
${ }^{*}$ Corresponding author, e-mail: fsy@sdu.edu.cn

(c) BME-PT
} 
hydrogels [13]. Reactive microgels have also been used as multifunctional crosslinkers for photopolymerized thin films or thermosets to improve their mechanical properties $[16,17]$. In addition to the direct polymerization method by adding the reactive microgels containing $\mathrm{C}=\mathrm{C}$ double bonds, the crosslinked structure can also be obtained by postcrosslinking of the polymers through irradiation, peroxide, drying, heating, and so on [18-21]. Amide groups of acrylamide can react with other groups [22]. The chemical reactions can occur between the amide groups of acrylamide (AM) and the hydroxymethyl groups of $N$-methylolacrylamide (NMA) at appropriate temperature [13]. Microgel composite (MC) hydrogels can be designed by the postcrosslinking method through heating the composite polymer with dispersed reactive microgels using the reaction between the microgels containing hydroxymethyl groups and the polymer linear chains containing amide groups. High mechanical strength hydrogels were obtained easily by the post-crosslinking method and the crosslinking did not affect the polymerization process. The method might open a new field to manufacture composite hydrogels with novel structures.

In this paper, AM and 2-Acrylamido-2-methylpropane sulfonic acid (AMPS) was used as main monomers. NMA was used as a functional monomer. First, the reactive microgels with hydroxymethyl groups were synthesized by inverse emulsion polymerization. Then, they were dispersed into the monomer solutions to synthesize composite polymers. Finally, the as-prepared composite polymers or partly evaporated composite polymers were heated to prepare MC hydrogels. Effects of the heating temperature, heating time and polymer water content on the swelling and mechanical properties of the MC hydrogels were investigated.

\section{Experimental Part}

\subsection{Materials}

AM (chemically pure, Dia-Nitrix. Co., Tokyo, Japan), AMPS (chemically pure, Shandong Lianmeng Chemical Group Co., Shouguang, China) and NMA (chemically pure, Shandong Zibo Xinye Chemical Co., Zibo, China) were used directly. The other reagents were all analytically pure, commercially available and were used without further purification. Distilled water was used in the whole experiments.

\subsection{Preparation of reactive microgels with hydroxymethyl groups}

Reactive microgels were prepared by inverse emulsion polymerization. AM solutions (98 $\mathrm{ml} 43 \mathrm{wt} \%$ ) and AMPS ( $5 \mathrm{~g})$ as monomers, NMA (5g) as a functional monomer, sorbitan monolaurate $(9 \mathrm{~g})$ and octylphenol ethoxylate $(2.5 \mathrm{~g})$ as emulsifiers and $N, N^{\prime}$-methylenebisacrylamide $(0.003 \mathrm{~g})$ as crosslinking agent were added into a $500 \mathrm{ml}$ round-bottomed three-neck flask with a refluxed condenser, a mechanical stirrer, a vent-plug and a thermometer. The resulting solution was stirred for $20 \mathrm{~min}$, and then cyclohexane $(110 \mathrm{ml})$ was added into the flask. After bubbled by nitrogen for $20 \mathrm{~min}$, the polymerization was initiated at $25^{\circ} \mathrm{C}$ using redox initiator of ammonium persulfate $(0.004 \mathrm{~g}$ in $1 \mathrm{ml}$ water $)$ and sodium bisulfite $(0.01 \mathrm{~g}$ in $1 \mathrm{ml}$ water). Nitrogen atmosphere was maintained throughout the polymerization. The microgel emulsions were obtained after $3 \mathrm{~h}$ reaction. First, $13 \mathrm{~g}$ of the microgel emulsions were precipitated by enough acetone to obtain microgel powder. Second, the microgel powder was dried at room temperature. Last, the dried powder was dispersed in $87 \mathrm{ml}$ distilled water to obtain dispersed solutions for further use.

\subsection{Preparation of composite polymer}

The composite polymer was prepared by solution polymerization. The above dispersed solution (13.6 g), distilled water (61.7 ml), AM (20.3 g) and AMPS ( $4 \mathrm{~g}$ ) were added into in a beaker. After nitrogen bubbling for $30 \mathrm{~min}$, the solution was initiated by redox initiator of ammonium persulfate $(0.004 \mathrm{~g}$ in $1 \mathrm{ml}$ water) and sodium bisulfite $(0.01 \mathrm{~g}$ in $1 \mathrm{ml}$ water) at $20^{\circ} \mathrm{C}$. The polymerization was carried out under airproof conditions for $5 \mathrm{~h}$ to obtain composite polymers and was cut into appropriate size to be used further. The as-prepared composite polymer has $75 \%$ water content. Polymer water content $x \%$ was calculated by the Equation (1):

$x[\%]=\frac{W-W_{\mathrm{pd}}}{W}$

where $W$ and $W_{\text {pd }}$ were the wet polymer weight and the corresponding dry polymer weight, respectively. 


\subsection{Preparation of MC hydrogels}

$\mathrm{MC}$ hydrogels were prepared by heating the as-prepared composite polymers or partly evaporated composite polymers with different water content under airproof conditions at 80 to $99^{\circ} \mathrm{C}$. When the polymer achieved the designed water content by evaporation, it was wrapped by plastic wrap and tape. It was held at room temperature for two days for uniform diffusion of the water within the whole polymer. Then, it was heated to prepare MC hydrogels. The MC hydrogels were referred to as MC-y$\mathrm{zh}$ and $\mathrm{MCx} \%-\mathrm{y}-\mathrm{zh}$ for heating the as-prepared composite polymers and the partly evaporated composite polymers, respectively. Here $x, y$ and $z$ were the partly evaporated polymer water content, heating temperature and the number of hours for heating time, respectively. The schematic illustration of preparing $\mathrm{MC}$ hydrogels by post-crosslinking is proposed in Figure 1a.

\subsection{Measurements of microgel particle size}

The particle size of the reactive microgels was detected by JEM-100CX II transmission electron microscopy (TEM, JEOL Ltd., Tokyo, Japan). TEM samples were prepared by placing a dilute drop of microgel particles onto a sample grid and allowing them natural drying in air. Dynamic light scattering measurements were performed on a Malvern Zetasizer 3000 instrument (Malvern Instruments Ltd., Malvern, UK) at $25^{\circ} \mathrm{C}$ and at a scattering angle of $90^{\circ}$ to obtain the swollen size of microgel particles in water.

\subsection{Measurements of the swelling properties}

The swelling experiments were performed by immersing the hydrogels in a large excess of water at room temperature to reach the swelling equilibrium. The swelling ratio was calculated by the Equation (2)

$Q=\frac{W_{\mathrm{e}}-W_{\mathrm{d}}}{W_{\mathrm{d}}}$

where $W_{\mathrm{e}}$ is the weight of the equilibrium swollen hydrogel and $W_{\mathrm{d}}$ is the corresponding dried hydrogel, respectively.

\subsection{Measurements of the degree of hydrolysis of the MC hydrogels}

During the heating process for preparing $\mathrm{MC}$ hydrogels, amide groups of acrylamide can hydrolyze at high temperature. The degree of hydrolysis $(z \%)$ of the amide groups was determined by the titration a)

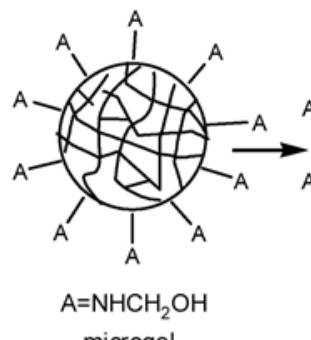

microgel

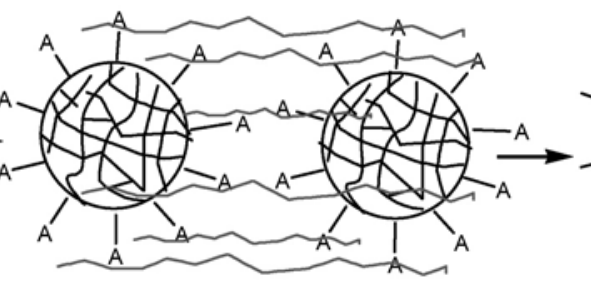

composite polymer

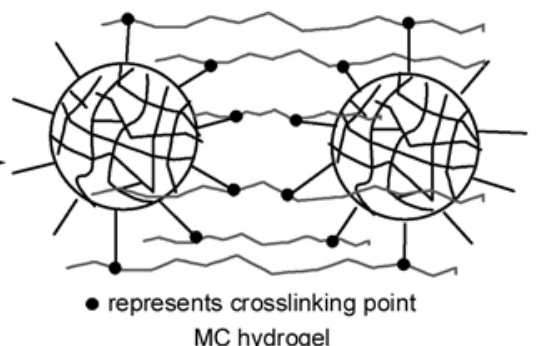

MC hydrogel<smiles>NC(=O)OCCOCCO</smiles><smiles>CCCCCCCCCCCCC(=O)NCO</smiles>

c)
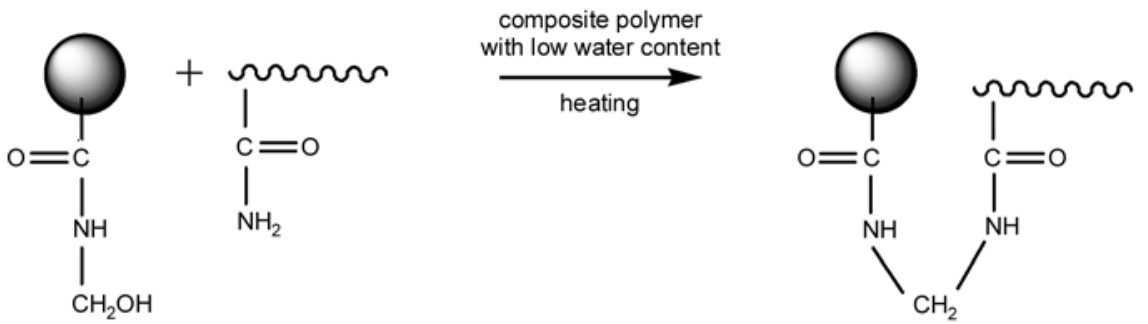

Figure 1. Schematic illustration of a MC hydrogel formation and crosslinking reactions between microgels and polymer matrix. (a) MC hydrogel formation, (b) hydrogen bond formation, (c) covalent bond formation 
method. The carboxyl groups produced by hydrolyzing were neutralized by excess $0.1 \mathrm{~mol} / 1$ standard solutions of sodium hydroxide. The remaining sodium hydroxide was titrated by $0.1 \mathrm{~mol} / 1 \mathrm{stan}-$ dard solution of hydrochloric acid. The Equation (3) was used to calculate the degree of hydrolysis of the product:

$z[\%]=\frac{\left(V_{1} C_{1}-V_{2} C_{2}\right)-\left(V_{01} C_{1}-V_{02} C_{2}\right)}{n} \cdot 100$

where $C_{1}$ and $C_{2}$ are the molar concentration of sodium hydroxide and hydrochloric acid standard solution, $V_{1}$ and $V_{2}$ are the total volume of sodium hydroxide solutions used for neutralization and the consumed volume of hydrochloric acid solution for hydrogels, $V_{01}$ and $V_{02}$ are the total volume of sodium hydroxide solutions used for neutralization and the consumed volume of hydrochloric acid standard solution for polymer without heating, $\mathrm{n}$ is the amount of the amide groups [mol], respectively. About $80 \mathrm{~g}$ swollen sample was used to measure the degree of hydrolysis. The amount of the amide groups was calculated by the corresponding acrylamide weight of the swollen sample. Three samples were tested and their results were averaged.

\subsection{Measurements of the mechanical properties of the MC hydrogels}

The mechanical properties of the hydrogels were measured by an electronic pulling tester (LR10KPLUS, $10 \mathrm{kN}$ Universal Materials Testing Machine, Lloyd Instruments, West Sussex, UK). The conditions were as follows: temperature $25^{\circ} \mathrm{C}$, sample water content $90 \%$, the sample size $8 \mathrm{~mm}$ (thickness) $\times 15 \mathrm{~mm}$ (width) $\times 60 \mathrm{~mm}$ (length), the gauge length $35 \mathrm{~mm}$ and crosshead speed $100 \mathrm{~mm} / \mathrm{min} .90 \%$ water content of the sample was prepared as follows. The hydrogel was immersed in water. Its water content was calculated by Equation (1) and controlled according to monitoring its weight. When its weights attained $90 \%$ water content, it was removed and put in plastic bags for uniform diffusion of the water within the whole gels. The tensile stress was calculated on the basis of the initial cross section of the specimen and the strain was defined as the change in the length with respect to the initial gauge length. Three samples were tested for each type of hydrogels and the data was averaged.

\section{Results and discussion}

\subsection{Influence of heating conditions on MC hydrogels prepared by heating the as-prepared composite polymers.}

The reactive microgels were prepared by inverse emulsion polymerization. The conversion of the monomers in microgel synthesis exceeded $99.5 \%$. It was determined by bromation of the residual double bond according to Liu et al. [23]. TEM was used to observe the microgel particle size. The microgel particles have sphere morphology. Their average dry particle size is about $100 \mathrm{~nm}$ (Figure 2). The average swollen size of the microgel particles is about $298 \mathrm{~nm}$ (Figure 3) and the polydispersity in microgel particle size was 0.449 , which was deter-

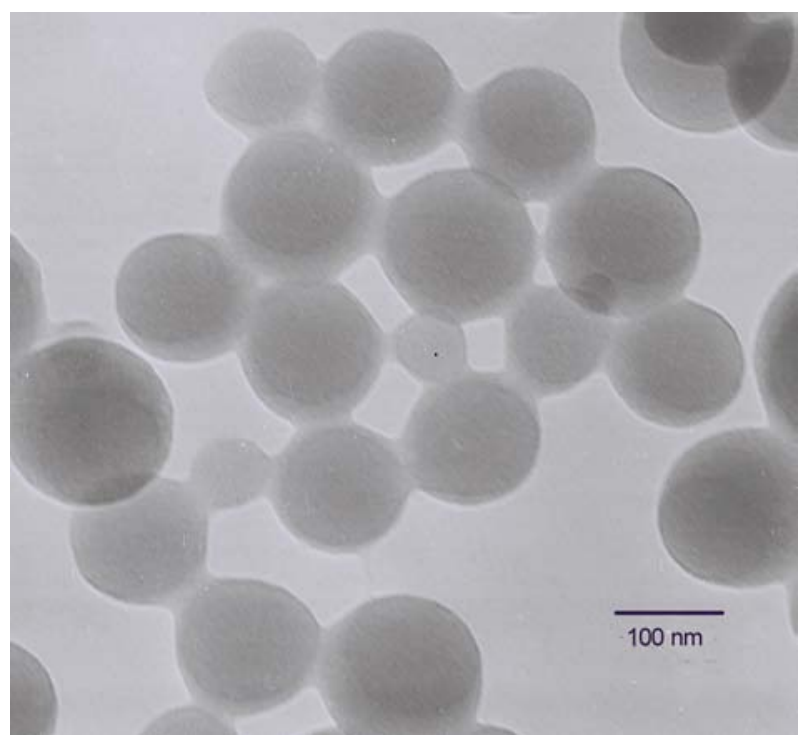

Figure 2. TEM images of the reactive microgel particles

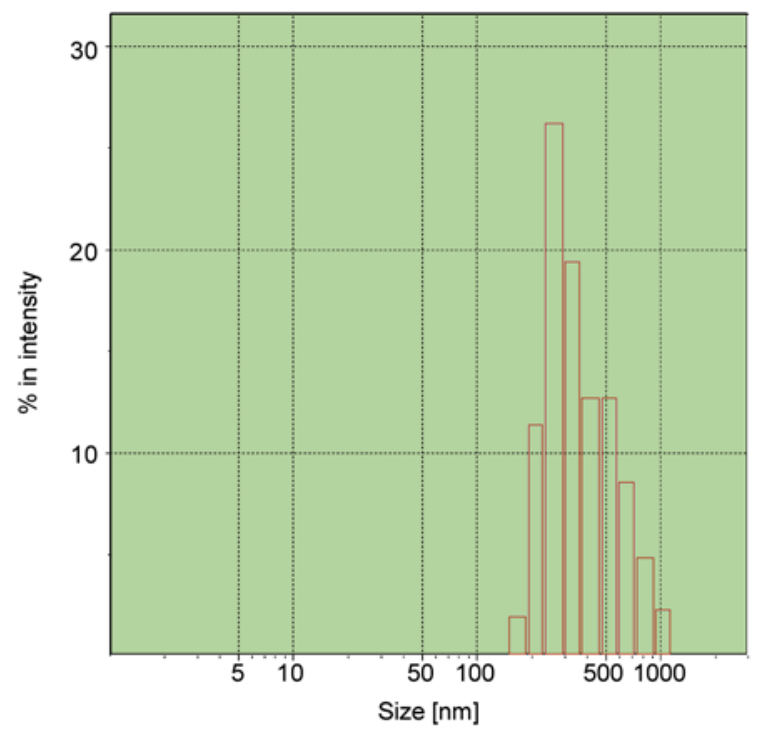

Figure 3. The swollen size of the microgels by dynamic light scattering measurements 
mined by dynamic light scattering. The reactive microgels contain hydroxymethyl groups, which were introduced into the microgel particles by using NMA as a functional monomer. The schematic illustration is shown in Figure 1. The hydroxymethyl groups can further react with other active groups such as amide groups and hydroxymethyl groups themselves at suitable temperature $[13,24]$. The schematic illustration of preparing $\mathrm{MC}$ hydrogels by post-crosslinking is proposed in Figure 1a. The reactive microgels containing hydroxymethyl groups act as potential post-crosslinking agents. The postcrosslinking reaction can occur between the hydroxymethyl groups and the functional groups of the polymer linear chains.

The composite polymer with embedded reactive microgels was prepared by solution polymerization. Reactive microgels containing hydroxymethyl groups were uniformly dispersed in the AM and AMPS solutions before polymerization. As shown in Table 1, the formation of MC hydrogels by direct heating the as-prepared composite polymer strongly depends on the heating temperature and time. The as-prepared composite polymer is still soluble when it is heated at $90^{\circ} \mathrm{C}$ less than $2 \mathrm{~h}$. When the composite polymer is heated at $90^{\circ} \mathrm{C}$ for more than $2 \mathrm{~h}$, it cannot dissolve in large excess water but swells in water and shows equilibrium swelling behavior. It suggests that the MC hydrogels are formed. As shown in Table 1, when the heating temperature decreases, it requires a longer heating time such as more than $3 \mathrm{~h}$ at $80^{\circ} \mathrm{C}$ to form hydrogels. It shows that the crosslinking reaction occurred more easily at high temperature for the as-prepared composite polymers.

In order to investigate the action of the reactive microgels, AM and AMPS copolymer without microgels was also prepared by solution polymerization under the same experimental conditions. As shown in Table 1, using the same heating time $5 \mathrm{~h}$ and the same temperature $90^{\circ} \mathrm{C}$ for the as-prepared AM and AMPS copolymer, which is named P-90$5 \mathrm{~h}$, the polymer fails to form a hydrogel and is still soluble. Compared with the composite polymer, the AM and AMPS copolymer is lacking of functional hydroxymethyl groups because it does not contain the reactive microgels. It indicates that the reactions of hydroxymethyl groups are the direct cause of the above MC hydrogel formation and the MC hydro-

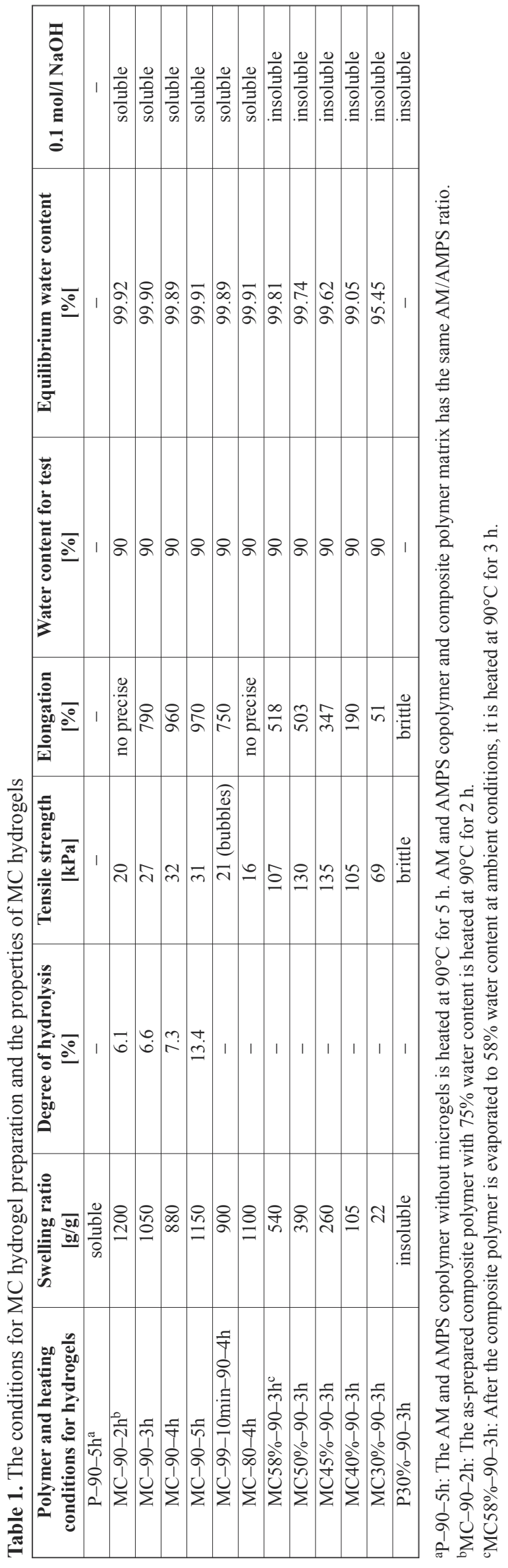


gels are crosslinked by microgel particles. Hydroxymethyl groups can form covalent bonds with amide groups [13] and hydrogen bonds with $\mathrm{C}=\mathrm{O}$ ester groups of amide (or carboxyl in case of hydrolysis). Either hydrogen bonds or covalent bonds between the hydroxymethyl groups and the amide groups of linear chains in the composite polymer can lead to crosslinking reaction for MC hydrogel formation as illustrated in Figure 1. To determine the hydrogen bonds or the covalent bonds crosslinking for forming the $\mathrm{MC}$ hydrogels, the swelling properties of the hydrogels in the $0.1 \mathrm{~mol} / 1$ sodium hydroxide solution was investigated [11]. As shown in Table 1, no matter how long heating for the asprepared composite polymers at $90^{\circ} \mathrm{C}$, the hydrogels are always soluble in the sodium hydroxide solution. Therefore, it is mainly due to the hydrogen bonds crosslinking rather than the covalent bonds crosslinking for forming the MC hydrogels.

The swelling character of the MC hydrogels from the as-prepared composite polymers were also investigated. As shown in Table 1, the swelling ratio decreases from 1200 to 880 when the heating time increases from 2 to $4 \mathrm{~h}$. Because the crosslinks of MC hydrogels are not points but nanoparticles, the swelling ratio of the MC hydrogels depends on the degree of crosslinks between microgels and the hydrogel matrix. According to the swelling theory [25], the hydrogel swelling ratio is inversely related to the crosslinking density. It indicates that the longer the reaction time, the more crosslinked bonds between the polymer matrix and the microgels are formed. However, when the heating time increases from 4 to $5 \mathrm{~h}$, the swelling ratio has a sudden increase from 880 to 1150 . This is inconsistent with the above crosslinking bonds forming character. Ionic carboxyl groups can be formed under high temperature because of the hydrolysis of amide groups. As shown in Table 1, the degree of hydrolysis of the amide groups increases from 7.3 to $13.4 \%$ when the heating time increases from 4 to $5 \mathrm{~h}$ at $90^{\circ} \mathrm{C}$. However, more carboxyl groups in the MC hydrogel hardly can significantly increase the swelling ratio. Increase of swelling ratio can be, probably, due to the self-reaction of methylol groups, as longer times at elevated temperature result in less crosslink degree of MC gels.
Mechanical properties were performed on the MC hydrogels with $90 \%$ water content. As shown in Table 1, for the above MC hydrogels from the asprepared composite polymers, both the heating temperature and heating time influence the mechanical properties. The tensile strength increases as the heating temperature increases from 80 to $90^{\circ} \mathrm{C}$. However, the tensile strength decreases as the temperature increases sequentially to $99^{\circ} \mathrm{C}$, because large numbers of bubbles generated in the hydrogel. The appropriate heating conditions for high tensile strength are $90^{\circ} \mathrm{C}$ and $4 \mathrm{~h}$. The tensile stress-strain curve of the MC-90-4h hydrogel is shown in Figure 4 . It has a high elongation of $960 \%$ and a high tensile strength of $32 \mathrm{kPa}$, which is much higher than that of the conventional $\mathrm{N}$-isopropylacrylamide hydrogels (tensile strength of $8-9 \mathrm{kPa}$ and elongation of 20-50\%) [9] or conventional AM hydrogels (tensile strength of $14 \mathrm{kPa}$ and elongation of $35 \%$ ) [26]. The excellent mechanical properties are attributed to the unique structure that the hydrogels are crosslinked by rubber microgels. However, the tensile strength is lower than that of the MC hydrogels crosslinked by covalent $\mathrm{C}-\mathrm{C}$ bonds reported by our groups [13]. As described above, the MC hydrogels by heating the as-prepared composite polymers are crosslinked by hydrogen bonds. This is consistent with the fact that hydrogen bonds are weaker than the covalent bonds. It suggests that the covalent bond crosslinking will benefit to improve the mechanical properties of MC hydrogels.

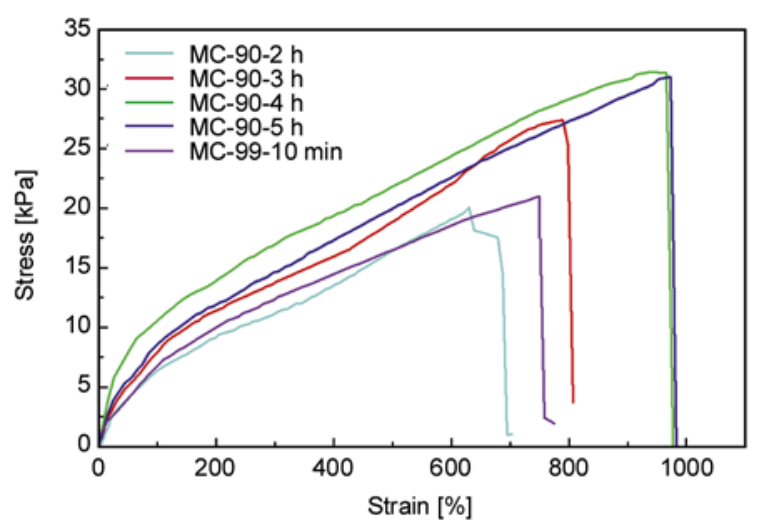

Figure 4. Stress-strain curves of the MC hydrogels at different heating conditions by heating the as-prepared composite polymers. (All hydrogel samples used for test has $90 \%$ water content.) 


\subsection{Effect of polymer water content on the MC hydrogels prepared by partly evaporated composite polymers}

When NMA was used as an efficient crosslinking agent, covalent cosslinking bonds could be formed by thermal activation at around $110^{\circ} \mathrm{C}$ after water was evaporated in the acrylate latex films [27-29]. After water was completely evaporated to form drying films, covalent cosslinking bonds could also be formed under ambient conditions [20]. It is suggested that decreasing the polymer water content is beneficial to the covalent bond formation when NMA is used as a crosslinking agent. When polymer water content decreases, the equilibrium water content decreases as shown in Table 1, which indicates the hydrogel has more crosslinking density. The as-prepared composite polymer has $75 \%$ water content. As described above, the hydrogels cannot form covalent crosslinking bonds between the microgels and the polymer matrix by directly heating the as-prepared composite polymer. Considering the influence of the water content on the covalent bond formation, partly evaporated composite polymer was used to prepare MC hydrogels.

First, natural drying polymer with near equilibrium water content (about 30\%) in natural environment was used. For MC30\%-90-3h hydrogels, after the polymer was evaporated to $30 \%$ water content, it was heated at $90^{\circ} \mathrm{C}$ for $3 \mathrm{~h}$. As shown in Table 1, it is insoluble and has low swelling ratio (about 22). It shows that the hydrogel is formed and has high crosslinking density. However, the hydrogel was very brittle and non-elastic. The tensile strength could not easy be obtained because it was difficult to clamp the hydrogel tightly enough for tensile tests and often broke before obtaining precise mechanical data. As shown in Table 1, although the tensile strength can reach $69 \mathrm{kPa}$, the elongation at break is only about $51 \%$ which is similar to the conventional hydrogel [9] and much lower than that of MC hydrogels prepared by the as-prepared composite polymers. As shown in Table 1, this hydrogel is insoluble in $0.1 \mathrm{~mol} / 1$ sodium hydroxide solutions. It indicates that covalent crosslinking bonds are formed in the above hydrogels. In order to investigate the reactions between the linear chains in the polymer matrix, the AM/AMPS polymer with the same water content $(30 \%)$ and heating conditions $\left(90^{\circ} \mathrm{C}\right.$ and $\left.3 \mathrm{~h}\right)$ as the above composite polymer was

investigated, which is named $\mathrm{P} 30 \%-90-3 \mathrm{~h}$. For $\mathrm{P} 30 \%-90-3 \mathrm{~h}$ polymers, it is also insoluble in $0.1 \mathrm{~mol} / 1$ sodium hydroxide solutions. It suggests that covalent crosslinking bonds occur between the linear poly(AM co AMPS) chains at $90^{\circ} \mathrm{C}$ and $3 \mathrm{~h}$ for polymer with $30 \%$ water content. Therefore, for MC30\%-90-3h, the hydrogel matrix between microgels also has crosslinking network structure similar to conventional hydrogels rather than linear chains. $\mathrm{NC}$ hydrogels with excellent mechanical properties cannot be obtained when the hydrogel matrix has crosslinked structure by adding $N, N^{\prime}$-methylenebisacrylamide [6]. For MC30\%-90-3h, excessive crosslinking of the linear polymer chains also form crosslinked hydrogel matrix causes the weak mechanical properties of the MC hydrogels.

As described above, the hydrogels cannot form covalent crosslinking bonds by heating the as-prepared composite polymer. However, the excessive drying of composite polymer will lead to conventional hydrogels with weak mechanical properties. That is, the polymer water content directly affects the hydrogel properties. For MC58\%-90-3h, after the composite polymer was evaporated to $58 \%$ water content, it was heated at $90^{\circ} \mathrm{C}$ for $3 \mathrm{~h}$. As shown in Table 1, the MC hydrogels is formed and their swelling ratio is 540 . They also cannot dissolve in $0.1 \mathrm{~mol} / 1$ sodium hydroxide solution. This MC hydrogels have excellent mechanical properties (Figure 5). It could withstand high levels of deforma-

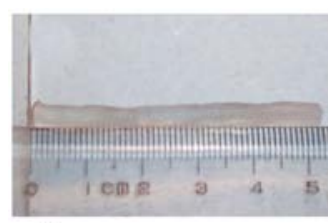

a)

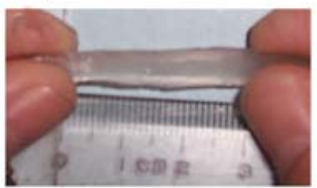

d)

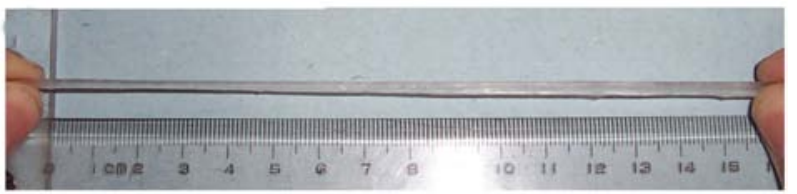

e)

Figure 5. Photographs demonstrated the mechanical properties of MC58\%-90-3h hydrogels. (a) and (d) original length, (b) bending, (c) knotting, (e) elongation, (f) recovered after elongation 
tion such as bending, torsion, knotting, and elongation. When it was allowed to recover after elongation, it could recover its original length. As shown in Table 1, it has a high elongation of $518 \%$, and high tensile strength of $107 \mathrm{kPa}$. This tensile strength is close to that of the NC hydrogels [6] and much higher than that of the hydrogels prepared by heating the as-prepared polymers or $30 \%$ water content polymers.

As the stress-strain curve shown in Figure 6 and Table 1, the tensile strength increases first and then decreases as polymer water content decreases. At $45 \%$ water content, the tensile strength can reach $135 \mathrm{kPa}$. However, the elongation always decreases as water content decreases. The composite polymer contains microgel and polymer matrix. For MC hydrogels, the crosslinkers are nanoparticles and the hydrogel matrix consists of linear polymer chains. This is different from the reported nanocomposite hydrogels that contain crosslinked hydrogel matrix by $N, N^{\prime}$-methylenebisacrylamide [30, 31]. Our groups reported that the strength of composite hydrogels with network hydrogel matrix is much lower than that of the MC hydrogels with linear hydrogel matrix [13]. The linear chains for hydrogel matrix between the particles can improve the mechanical properties of the composite hydrogels. For this reason, the mechanical properties of crosslinked hydrogels depend on the matrix, the microgels and the degree of crosslinks between the matrix and microgels. As the polymer water content decreases, the polymer chain length between the microgels decreases because the distances between the microgels decrease [6]. Since the decrease of

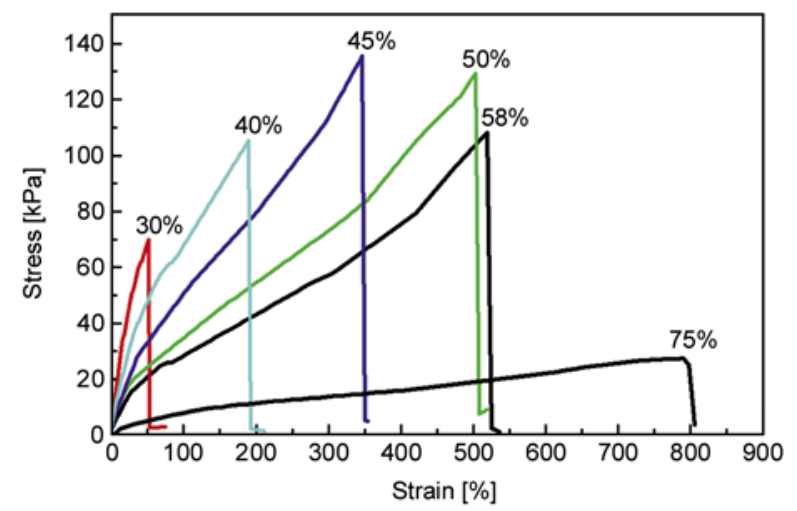

Figure 6. Stress-strain curves of $\mathrm{MCx} \%-90-3 \mathrm{~h}$ hydrogels. ( $x \%$ making on the curves is the polymer water content for preparing hydrogels. All hydrogel samples used for test has $90 \%$ water content.) distance between microgels during the synthesis is supposed to cause the higher crosslink degree of MC hydrogels, which leads to the decrease of the tensile elongation. As polymer water content decreases, the more covalent bond formation between the microgel and matrix will lead to higher tensile strength. However, with further decrease of polymer water content, the hydrogel matrix is also crosslinked and is similar to traditional hydrogels because of the reaction between the linear chains, which leads to the decreasing of the tensile strength. As described above, the tensile strength and the elongation can easily be adjusted by the composite polymer water content. As shown in Figure 6, for composite polymer with $50 \%$ water content, both tensile strength and elongation has high values with $130 \mathrm{kPa}$ of tensile strength and 503\% elongation.

\section{Conclusions}

The MC hydrogels have been successfully prepared by post-crosslinking method by heating the composite polymer. The MC hydrogels prepared at suitable conditions has excellent mechanical properties such as high elasticity, high tensile strength, and high elongation. This post-crosslinking method might open a new convenient method to manufacture soft and wet hydrogels with high mechanical properties.

For MC-90 hydrogels prepared by heating the asprepared composite polymers with $75 \%$ water content, the formation of hydrogels and their properties strongly depend on the heating conditions. More than $2 \mathrm{~h}$ is required at $90^{\circ} \mathrm{C}$. As temperature decreases, more time are required to form hydrogels. With the heated temperature increasing, the swelling ratio decreases first and then increases. However, it is soluble in $0.1 \mathrm{~mol} / 1 \mathrm{NaOH}$ solution. Hydrogen bonds are the main crosslinking bonds. For MC90-4h, the MC hydrogels have a tensile strength of $32 \mathrm{kPa}$, which is higher than that of the conventional hydrogels but lower than that of the MC hydrogels crosslinked by covalent bonds.

The mechanical properties and swelling ratio of MC hydrogels are influenced by the polymer water content. When the polymer water content is lower than $58 \%$, the MC hydrogel cannot dissolve in $0.1 \mathrm{~mol} / \mathrm{l} \mathrm{NaOH}$ solutions. For MC hydrogels prepared by heating the partly evaporated composite polymers, as water content decreases, the tensile 
strength increases first and then decreases. However, the elongation always decreases as water content decreases. The tensile strength and the elongation can be adjusted according to the polymer water content. When the composite polymer with $50 \%$ water content is heated at $90^{\circ} \mathrm{C}$ for $3 \mathrm{~h}$, the hydrogels have high tensile strength of $130 \mathrm{kPa}$ and tensile elongation of $503 \%$. When the polymer water content is $30 \%$, the hydrogels have low swelling ratio and weak mechanical properties similar to the conventional hydrogels.

\section{Acknowledgements}

This study was supported by Shandong Province Excellent Middle-Aged and Young Scientist Research Incentive Foundation (Grant No. 2008BS04008) and Independent Innovation Foundation of Shandong University (2010TS099).

\section{References}

[1] Bohidar H. B., Dubin P., Osada Y.: Polymer gels: Fundamentals and applications. ACS Symposium Series, San Francisco (2002).

[2] Wang Q., Mynar J. L., Yoshida M., Lee E., Lee M., Okuro K., Kinbara K., Aida T.: High-water-content mouldable hydrogels by mixing clay and a dendritic molecular binder. Nature, 463, 339-343 (2010). DOI: 10.1038/nature08693

[3] Okumura Y., Ito K.: The polyrotaxane gel: A topological gel by figure-of-eight cross-links. Advanced Materials, 13, 485-487 (2001).

DOI: $10.1002 / 1521-4095(200104) 13: 7<485:: A I D-$ ADMA485>3.0.CO;2-T

[4] Gong J. P., Katsuyama Y., Kurokawa T., Osada Y.: Double-network hydrogels with extremely high mechanical strength. Advanced Materials, 15, 11551158 (2003). DOI: $10.1002 /$ adma.200304907

[5] Webber R. E., Creton C., Brown H. R., Gong J. P.: Large strain hysteresis and mullins effect of tough double-network hydrogels. Macromolecules, 40, 29192927 (2007). DOI: $10.1021 / \mathrm{ma} 062924 \mathrm{y}$

[6] Haraguchi K., Takehisa T.: Nanocomposite hydrogels: A unique organic-inorganic network structure with extraordinary mechanical, optical, and swelling/deswelling properties. Advanced Materials, 14, 11201124 (2002).

DOI: $10.1002 / 1521-4095(20020816) 14: 16<1120:: A I D-$ ADMA1120>3.0.CO;2-9

[7] Haraguchi K., Farnworth R., Ohbayashi A., Takehisa T.: Compositional effects on mechanical properties of nanocomposite hydrogels composed of poly $(\mathrm{N}, \mathrm{N}-$ dimethylacrylamide) and clay. Macromolecules, 36, 5732-5741 (2003).

DOI: $\underline{10.1021 / \mathrm{ma} 034366 \mathrm{i}}$
[8] Haraguchi K., Takehisa T., Fan S.: Effects of clay content on the properties of nanocomposite hydrogels composed of poly(N-isopropylacrylamide) and clay. Macromolecules, 35, 10162-10171 (2002).

DOI: $10.1021 / \mathrm{ma} 021301 \mathrm{r}$

[9] Haraguchi K., Li H-J.: Mechanical properties and structure of polymer-clay nanocomposite gels with high clay content. Macromolecules, 39, 1898-1905 (2006).

DOI: $10.1021 / \mathrm{ma} 052468 \mathrm{y}$

[10] Tanaka Y., Gong J. P., Osada Y.: Novel hydrogels with excellent mechanical performance. Progress in Polymer Science, 30, 1-9 (2005).

DOI: 10.1016/j.progpolymsci.2004.11.003

[11] Huang T., Xu H. G., Jiao K. X., Zhu L. P., Brown H. R., Wang H. L.: A novel hydrogel with high mechanical strength: A macromolecular microsphere composite hydrogel. Advanced Materials, 19, 1622-1626 (2007).

DOI: $10.1002 /$ adma.200602533

[12] Lu X., Hu Z., Gao J.: Synthesis and light scattering study of hydroxypropyl cellulose microgels. Macromolecules, 33, 8698-8702 (2000).

DOI: $10.1021 / \mathrm{ma} 000776 \mathrm{k}$

[13] Qin X. P., Zhao F., Liu Y. K., Wang H. Y., Feng S. Y.: High mechanical strength hydrogels preparation using hydrophilic reactive microgels as crosslinking agents. Colloid and Polymer Science, 287, 621-625 (2009). DOI: 10.1007/s00396-009-2018-Z

[14] Wu Y. T., Zhou Z., Fan Q. Q., Chen L., Zhu M. F.: Facile in-situ fabrication of novel organic nanoparticle hydrogels with excellent mechanical properties. Journal of Materials Chemistry, 19, 7340-7346 (2009). DOI: 10.1039/b909125d

[15] Schexnailder P., Schmidt G.: Nanocomposite polymer hydrogels. Colloid and Polymer Science, 287, 1-11 (2009).

DOI: $10.1007 / \mathrm{s} 00396-008-1949-0$

[16] Valette L., Pascault J-P., Magny B.: Use of acrylic functionalized (meth)acrylic cross-linked polymer microparticles in photopolymerized acrylic films. Macromolecular Materials and Engineering, 288, 642657 (2003).

DOI: $10.1002 /$ mame.200300012

[17] Valette L., Pascault J-P., Magny B.: Use of functional (meth)acrylic cross-linked polymer microparticles as toughening agents for epoxy/diamine thermosets. Macromolecular Materials and Engineering, 288, 867874 (2003).

DOI: $10.1002 /$ mame.200300041

[18] Liu H. Q., Zhen M., Wu R. H.: Ionic-strength- and pHresponsive poly[acrylamide-co-(maleic acid)] hydrogel nanofibers. Macromolecular Chemistry and Physics, 208, 874-880 (2007).

DOI: $10.1002 / \mathrm{macp} .200600632$ 
[19] Abbas L., Bouquey M., Flat J. J., Muller R.: New method for post-processing crosslinking reaction. European Polymer Journal, 44, 1238-1246 (2008).

DOI: 10.1016/j.eurpolymj.2008.01.018

[20] Zhou J., Wang G., Marquez M., Hu Z.: The formation of crystalline hydrogel films by self-crosslinking microgels. Soft Matter, 5, 820-826 (2009).

DOI: $10.1039 / \mathrm{b} 814830 \mathrm{a}$

[21] Zhang W., Liu Y., Zhu M., Zhang Y., Liu X. L., Jiang Y. M., Chen Y. M., Kuckling D., Adler H-J. P.: Surprising conversion of nanocomposite hydrogels with high mechanical strength by posttreatment: From a low swelling ratio to an ultrahigh swelling ratio. Journal of Polymer Science Part A: Polymer Chemistry, 44, 6640-6645 (2006).

DOI: $10.1002 /$ pola.21749

[22] Fong D. W., Kowalski D. J.: An investigation of the crosslinking of polyacrylamide with formaldehyde using 13C nuclear magnetic resonance spectroscopy. Journal of Polymer Science Part A: Polymer Chemistry, 31, 1625-1627 (1993).

DOI: 10.1002/pola.1993.080310633

[23] Liu X., Xiang S., Yue Y., Su X., Zhang W., Song C., Wang P.: Engineering particle technology preparation of poly(acrylamide-co-acrylic acid) aqueous latex dispersions using anionic polyelectrolyte as stabilizer. Colloids and Surfaces A: Physicochemical and Engineering Aspects, 311, 131-139 (2007).

DOI: $10.1016 /$ j.colsurfa.2007.06.007

[24] Lu S. J., Yao K., Lin S., Cao D., Chen Y.: Study on preparation and thickening efficiency of an inverse emulsion of anionic starch-graft-polyacrylamide. Starch/ Stärke, 55, 518-523 (2003). DOI: $10.1002 /$ star.200300166

[25] Flory P. J.: Principles of polymer chemistry. Cornell University Press, New York (1953).
[26] Zhu M. F., Liu Y., Sun B., Zhang W., Liu X. L., Yu H., Zhang Y., Kuckling D., Adler H. J. P.: A novel highly resilient nanocomposite hydrogel with low hysteresis and ultrahigh elongation. Macromolecular Rapid Communication, 27, 1023-1028 (2006).

DOI: $10.1002 /$ marc.200600159

[27] Chen Y., Zhang C., Wang Y., Cheng S., Chen P.: Study of self-crosslinking acrylate latex containing fluorine. Journal of Applied Polymer Science, 90, 3609-3616 (2003).

DOI: 10.1002/app.13087

[28] Chen S-I., Sheu Y-L., Sheu J-L., Lee C-T., Lin J. S.: Morphology of perfluoroalkylacrylate/stearyl methacrylate polymers and their effect on water/oil repellency. Journal of Applied Polymer Science, 63, 903-909 (1997).

DOI: 10.1002/(SICI)1097-4628(19970214)63:7<903:: AID-APP10>3.0.CO;2-S

[29] Wyss A., Von Stockar U., Marison I. W.: Production and characterization of liquid-core capsules made from cross-linked acrylamide copolymers for biotechnological applications. Biotechnology and Bioengineering, 86, 563-572 (2004).

DOI: $10.1002 /$ bit.20050

[30] Musch J., Schneider S., Lindner P., Richtering W.: Unperturbed volume transition of thermosensitive poly(N-isopropylacrylamide) microgel particles embedded in a hydrogel matrix. Journal of Physical Chemistry B, 112, 6309-6314 (2008).

DOI: $10.1021 / \mathrm{jp} 711939 \mathrm{v}$

[31] Salvati A., Söderman O., Lynch I.: Plum-pudding gels as a platform for drug delivery: Understanding the effects of the different components on the diffusion behavior of solutes. Journal of Physical Chemistry B, 111, 7367-7376 (2007). DOI: $\underline{10.1021 / j p 069051 \mathrm{u}}$ 